\title{
Type VI secretion system: a modular toolkit for bacterial dominance
}

\author{
Biswanath Jana ${ }^{1} \&$ Dor Salomon*,1 (iD) \\ ${ }^{1}$ Department of Clinical Microbiology \& Immunology, Sackler Faculty of Medicine, Tel Aviv University, Tel Aviv 6997801, Israel \\ *Author for correspondence: dorsalomon@mail.tau.ac.il
}

Bacteria use toxin delivery systems, such as the type VI secretion system (T6SS), to antagonize competitors. The T6SS transports toxins, called effectors, directly into recipient cells. In the absence of cognate immunity proteins that protect against kin-intoxication, these effectors target conserved and essential cell components resulting in growth arrest or cell death. Here, we focus on antibacterial T6SS effectors and explore their different activities, modes of delivery, and the domains and proteins that are associated with them to provide a modular and dynamic toxin arsenal. We conclude that these natural machines present a lucrative pool and platform for future antibacterial treatments.

First draft submitted: 26 June 2019; Accepted for publication: 29 October 2019; Published online: 13 November 2019

Keywords: antibacterial • bacterial competition • effector $\bullet$ secretion • toxin $\bullet$ type VI secretion system

Bacteria often live in large communities, either homogeneous or heterogeneous in composition [1]. Residents of such communities know how to converse (e.g., quorum sensing), but also how to quarrel. In recent years, we have witnessed the discovery of many bacterial weapons that are used in wars for dominance. Such antibacterial weapons utilize proteinaceous arsenals, referred to as toxins or effectors, that include bacteriocins [2], contact-dependent inhibition systems mediated via type I secretion system [3] or type V secretion system [4], type VI secretion system (T6SS) [5,6] and other phage-like contractile nanomachines generally referred to as tailocins [7], type VII secretion system [8] and even a subset of the otherwise virulence type IV secretion system [9]. Above all others, T6SS stands out with its breadth of activities and toxins that have been discovered.

T6SS is a multiprotein apparatus that is widespread in Gram-negative bacteria [10]. It delivers proteins, called effectors, directly into neighboring cells in a contact-dependent manner. Structurally, T6SS is similar to a contractile phage tail [11]. A membrane-spanning complex recruits a baseplate complex to the site of T6SS biogenesis on the cell envelope. The baseplate provides the origin for the biogenesis of a tail complex that is composed of a contractile outer tube, called sheath, and an inner tube. The inner tube, which is engulfed by the sheath, is propelled out of the cell upon activation of the system and contraction of the sheath. The secreted inner tube is composed of stacked hexameric rings of $\mathrm{Hcp}$ (hemolysin co-regulated protein), that are capped by a spike complex; the spike is a trimer of VgrG (valine-glycine repeat protein G) proteins that are sharpened by a PAAR (proline-alanine-alanine-arginine) repeat-containing protein. A detailed discussion of the structure and biogenesis of T6SS is beyond the scope of this review, and we refer the reader to a recent review on this topic [11].

T6SS was initially described as a protein secretion system in Rhizobium leguminosarum [12], Edwardsiella tarda [13], Vibrio cholerae [5] and Pseudomonas aeruginosa [6], where it was identified as a determinant that plays a role in interactions with eukaryotes. However, since the discovery of antibacterial T6SS effectors made by Hood et al. [14], it has become apparent that most T6SSs play a role in interbacterial competition. Accordingly, many T6SS effectors were shown to mediate antibacterial toxicity. To protect themselves from self- or kin-intoxication, bacteria encode cognate immunity proteins that antagonize the toxic activities of T6SS effectors, usually via direct interaction between the immunity protein and the toxin domain of the effector [14,15]. The effectors decorate the secreted inner tube and spike, either as domains fused to the secreted components Hcp, VgrG or PAAR-containing protein, or as separate proteins that are loaded onto them (named 'cargo effectors'). Penetration of the secreted tube into a neighboring cell enables the deployment of the effectors, which can then mediate the toxic activities of T6SS. In 


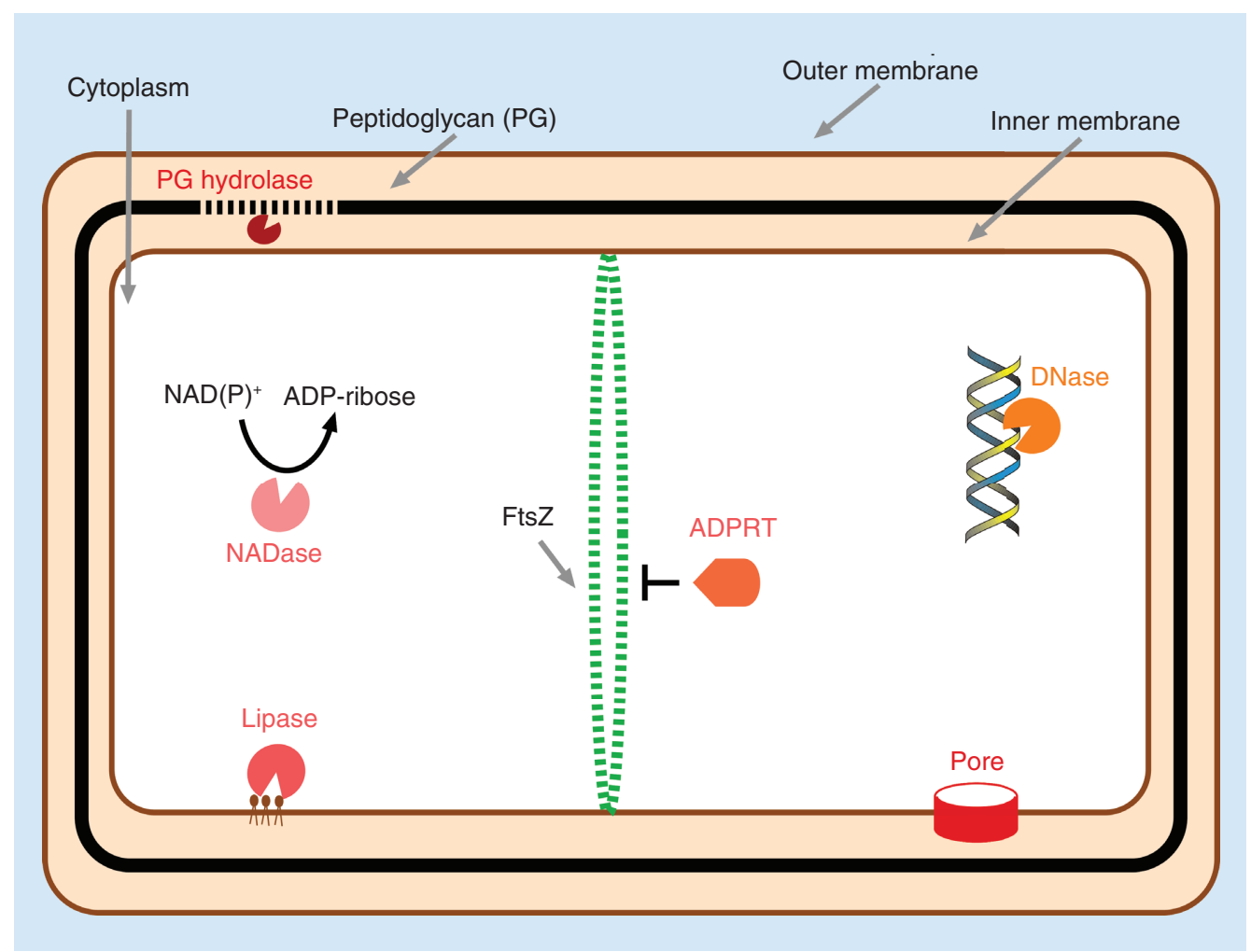

Figure 1. Antibacterial type VI secretion system effectors target essential cell components and processes. Illustration of antibacterial type VI secretion system effectors, their targets and activities (denoted in red) inside a Gram-negative bacterial cell. ADPRT: ADP-ribosyltransferase; PG: Peptidoglycan in cell wall; NADase: NAD(P)+ hydrolase.

this review, we will present and discuss recent advances and discoveries in the field of antibacterial T6SS effectors, their activities, targets, mode of delivery and mobility that enables the diversification of effector arsenals.

\section{Antibacterial T6SS effectors: activities \& targets}

T6SSs of many bacteria were shown to possess a repertoire of effectors. These T6SSs can thus deliver multiple effectors with diverse targets into competing cells. Most effectors have been shown to target vital bacterial cell components, while others can target conserved and essential bacterial proteins. Notably, various toxin domains remain with either putative or unexplored functions and targets (e.g., deaminase and protease) [16,17]. In this review, we will focus on T6SS effectors with validated antibacterial activities (Figure 1).

\section{Cell wall}

The cell wall is a bacterial outer barrier. It provides strength and protection against outside insults. Therefore, it is a lucrative target for antibacterial warfare. Indeed, animals use proteins that target the bacterial cell wall, such as lysozyme [18], to fight invading bacteria. It is, therefore, not surprising that bacteria also developed weapons to target the cell wall of competitors. The main target of such antibacterial proteins is the peptidoglycan. The peptidoglycan (also named murein) is a mesh-like layer composed of sugar (glycan) strands that are interlinked by amino acids (peptides). The glycan strands are made of alternating $\beta$-1,4-linked $N$-acetylmuramic acid (MurNAc) and $\mathrm{N}$-acetylglucosamine (GlcNAc). MurNAc are linked via an amide bond to short peptide stems, which are themselves cross-linked to bridge the glycan strands [19].

In 2010, Hood et al. discovered that a T6SS of $P$. aeruginosa delivers at least three effectors with antibacterial activities into neighboring cells [14]. Two of these effectors, named Tse1 (type six exported 1) and Tse3, were later shown to exert their antibacterial activity by cleaving the peptidoglycan of neighboring cells [20]. To mediate their toxic activity, the effectors had to be delivered to the periplasm where the peptidoglycan resides. Tse 1 was shown to be an amidase that cleaved bonds between the amino acids in the peptidoglycan, while Tse3 was found to have 
a lysozyme-like muramidase activity as it cleaved the glycan backbone between MurNAc and GlcNAc [20]. Since these discoveries, several families of T6SS effectors that target peptidoglycan have been described.

The first superfamily of T6SS peptidoglycan hydrolases was reported by Russell et al. Its members were named Tae (type VI amidase effector) after their amidase activities, cleaving peptidoglycan within the peptide stems and cross-links [15]. Using a heuristic approach and a set of parameters aimed at identifying amidase T6SS effectors, the authors revealed a widespread superfamily of amidases that segregated into four families, named Tae1-4, based on primary sequence homology [15]. All four families contained the conserved catalytic cysteine and histidine residues typical of amidases [21]. Notably, the above-mentioned Tse1 was reported as a member of the Tae1 family. Representatives of each Tae family were tested and shown to prefer different bonds within the peptidoglycan peptide stem for cleavage. Tae 1 and Tae 4 cleaved the bond between the D-Glu and m-DAP residues, while Tae 2 and Tae3 cleaved the bond between m-DAP and D-Ala [15]. In addition to the Tae1-4 families, a family named TaeX was recently added to the Tae superfamily. The only representative studied from this family, VT1 from Escherichia coli, was shown to possess an Amidase_2 domain and to cleave the amide bond between the MurNAc glycan and the first residue of the peptide stem, L-Ala [22]. Additional members of the Tae superfamily, such as the Serratia marcescens Ssp1 and Ssp2 effectors [23,24], have also been demonstrated to be T6SS effectors with amidase activities.

Another broadly distributed superfamily of T6SS effectors that target the peptidoglycan is Tge (type VI glycoside hydrolase effector) [25]. The Tge superfamily, comprised of three families named Tge1-3, was also found using an informatics approach similar to the one described above for finding the Tae superfamily. This search yielded two of the Tge families, while the aforementioned Tse3 muramidase was used to define the third Tge family. Tge members have a lysozyme-like fold, and were shown to cleave the glycoside bond between MurNAc and GlcNAc using a conserved catalytic glutamate residue [25]. It remains to be seen whether the preference of various effectors toward different bonds results from the prevalence of these bonds in the peptidoglycan of competitors in the natural environment.

\section{Nucleic acids}

Nucleases are an abundant and widespread class of antibacterial toxins. Several families of putative nuclease toxins have been predicted to associate with T6SSs [26], yet only a few have been experimentally validated. While both DNase and RNase effectors have been proposed [16,17,26-28], only DNase activity was demonstrated for T6SS nuclease effectors to date. Most of the validated DNase T6SS effectors contain toxin domains of the HNH (histidine, asparagine, histidine)-endonuclease superfamily [29]. In 2013, Koskiniemi et al. found that the antibacterial effectors RhsA and RhsB, of the soft rot pathogen Dickeya dadantii, contain C-terminal NS_2 endonuclease and $\mathrm{HNH}$-endonuclease domains, respectively. The authors used fluorescence microscopy to demonstrate that these effectors target and degrade DNA in recipient prey cells during bacterial competition [30]. The S. marcescens T6SS effector Rhs2 was also shown to harbor a C-terminal partial $\mathrm{HNH}$-endonuclease domain that mediated DNA degradation in vitro [31]. T6SS effectors containing AHH (alanine, histidine, histidine) or GHH2 (glycine, histidine, histidine) domains, belonging to the HNH superfamily, were also shown to function as DNases by either in vitro or in vivo assays. These include, for example, Rhs2 of Acinetobacter baumannii [32], Hcp-fused effectors of $E$. coli [33] and the PAAR-containing P. aeruginosa effector Tse7 [34].

Another family of T6SS DNase effectors was found in the plant pathogen Agrobacterium tumefaciens. Ma et al. identified two T6SS effectors, Tde1 (type VI DNase effector) and Tde2, that contained C-terminal toxin_43 domains belonging to a superfamily that shared a catalytic HxxD motif [29]. Although the toxin_43 domain was predicted to have RNase activity [17], the authors did not detect RNase activity in vitro but rather they found $\mathrm{Mg}^{2+}$-dependent DNase activity [29].

Recently, a family of DNase T6SS effectors named PoNe (polymorphic nuclease effectors) was reported [28]. PoNe belongs to a highly diverse superfamily of PD-(D/E)xK phosphodiesterases [35]. PoNe-containing effectors were shown to mediate DNA degradation both in vitro and in vivo during bacterial competition [28]. Notably, PoNe DNases share similar features, including a putative catalytic motif, with other predicted T6SS toxin domains annotated as restriction endonuclease like (REase) [26,28]. While these REases were previously predicted to possess DNase activity [26], this activity was not experimentally validated.

\section{Membranes}

Two main classes of effectors were shown to target bacterial membranes: pore-forming toxins and phospholipases. The first pore-forming T6SS effector to be described was VasX from V. cholerae, which contains predicted C- 
terminal transmembrane helices. Upon delivery to the periplasm, VasX compromised the integrity of the inner membrane as evident by loss of membrane potential and by increased cellular permeability to the intercalating dye, propidium iodide [36]. These phenotypes resembled those caused by pore-forming colicins [37]. Recently, LaCourse et al. identified the $P$. aeruginosa Tse4, a T6SS effector that contains predicted transmembrane domains and localizes to the membrane, as another pore-forming toxin. However, unlike VasX, Tse4 pores allow ions, but not larger molecules such as propidium iodide, to cross [38]. Other T6SS effectors were also suggested to be pore-forming toxins based on homology to pore-forming colicins and the presence of transmembrane helices at their predicted C-terminal toxin domains; their activities have yet to be demonstrated [27,39].

Several families of T6SS phospholipase effectors have been described to date. In 2013, Russell et al. used an informatics approach and analyzed predicted T6SS-associated lipases [40]. Five widespread, divergent families of lipase effectors, named Tle1-5 (Type VI lipase effector), were uncovered. Tle1-4 share a GXSXG conserved motif and a Ser-Asp-His catalytic triad, while Tle5 share a dual HXKXXXXD motif [40]. Phospholipase activity was demonstrated in vitro for Tle1 (phospholipase $A_{2}$ ), Tle2 (phospholipase $A_{1}$ ) and Tle5 (phospholipase D) family members. Tle5 was also shown to affect membrane phospholipid composition in vivo, resulting in cell death by targeting phosphatidylethanolamine, which leads to accumulation of phosphatidic acid [40]. A DUF2235-containing effector that belongs to the Tle1 family was later also shown to possess phospholipase $A_{1}$ and phospholipase $A_{2}$ activity [41].

\section{Energy balance}

Another mechanism used by T6SS effectors is manipulation of the energy balance in the prey cell. Effectors belonging to two phylogenetically distinct families were shown to exert their toxicity by depleting bacterial cells of the co-factors $\mathrm{NAD}^{+}$and $\mathrm{NADP}^{+}$(thus they are called NADases), thereby inhibiting both anabolic and catabolic processes [42,43]. The first NADase to be discovered was the P. aeruginosa T6SS effector Tse6 [42]. The Tse6 C-terminal toxin domain structurally resembled mono-ADP ribosyltransferase toxins, which use $\mathrm{NAD}^{+}$as their ADP donor, yet it differed in activity. Instead of using $\mathrm{NAD}^{+}$to modify a target protein, Tse6 hydrolyzed $\mathrm{NAD}^{+}$and $\mathrm{NADP}^{+}$, both in vitro and in cells, resulting in growth arrest [42]. Subsequently, Tang et al. identified the Pseudomonas protegens effector PFL_6209 as a NADase. While PFL_6209 is structurally similar to Tse6, there are some differences between the two NADases [43]. Therefore, Tse6 and PFL_6209 were defined as the founding members of two NADase effector families named Tne1 and Tne2 (type VI secretion NADase effector family 1 and 2), respectively [43].

\section{Post-translational modifications}

Most T6SS effectors described to date target vital bacterial cell components and processes such as nucleic acids, membranes, cell wall or energy balance, as described above. Nevertheless, a recent example revealed that T6SS effectors also target specific bacterial proteins. Tre1 (type VI secretion ADP-ribosyltransferase effector 1), a Serratia proteamaculans effector, was the first example of a T6SS effector that exerts its antibacterial toxicity by a posttranslational modification. Tre1 was shown to use its ADP-ribosyltransferase activity to post-translationally modify FtsZ, an essential protein that is critical for cell division, thus blocking its ability to polymerize. This modification resulted in cell division arrest. Interestingly, Tre1's cognate immunity, Tri1, antagonized this toxicity not only by directly occluding the toxin active site as other immunity proteins do, but also by catalytically cleaving the ADP-ribose off the target protein [44].

\section{Trans-kingdom effectors}

Several antibacterial T6SSs were shown to target not only competing bacteria, but also eukaryotic cells. Thus, they were termed trans-kingdom effectors. For example, the $V$. cholerae pore-forming effector VasX was originally identified as a virulence toxin against grazing amoeba [45], but was later shown to also mediate interbacterial competition [36]. In addition, the P. aeruginosa phospholipase D effectors, PldA and PldB, were shown to contribute to the pathogen's ability to invade epithelial cells by manipulating the PI3K-AKT signaling pathway, in addition to their role in interbacterial competition [46]. A third P. aeruginosa effector, TplE (belonging to the Tle4 family), was also shown to be a trans-kingdom phospholipase. TlpE inhibited bacterial growth and also enhanced autophagic flux in eukaryotic cells by activating the unfolded protein response via the IRE1 $\alpha$-XBP1 pathway [47]. While only pore-forming and lipase effectors were described as trans-kingdom effectors to date, it is likely that effectors employing other mechanisms of action will be found as being trans-kingdom effectors in the near future. It is 
plausible that effectors that target nucleic acids (DNases or RNases), the cell's energy balance (NADases) or other proteins (ADP-ribosyltransferases and proteases) in bacteria, will also target similar cell components found in eukaryotic cells.

\section{Effector synergism \& efficacy}

Why do bacteria carry diverse repertoires of multiple T6SS effectors? Delivering multiple effectors into competitors could possibly be a strategy to minimize the chances of encountering an immune competitor. However, another explanation can be that effectors have different efficacies under changing environments, and hence relying on a single effector could prove risky. Moreover, effectors could operate in synergy inside the prey, thus requiring a variety of effector combinations. In a recent report, LaCourse et al. introduced the concepts of conditional toxicity and synergism between T6SS effectors [38]. The authors tested $P$. aeruginosa T6SS effectors under different conditions such as salinity, temperature or $\mathrm{pH}$, and found that some effectors have significantly altered efficacies under tested conditions [38]. In addition, the authors found that the cumulative activities of several effector pairs exceeded the sum of their individual toxicity. This efficacy of synergistic effect was occasionally also dependent on external conditions [38]. Several questions arise from these discoveries: how do different repertoires influence target range of T6SSs?; does a given bacterium deliver all of its encoded effectors simultaneously, or are they delivered as separate groups that change based on varying external conditions?; do isolates of the same species that harbor different effector arsenals use their T6SS to target specific competitors in their local environment?; what mechanisms are used to diversify effectors repertoires (see discussion on mobility of effectors below)?

\section{Mechanisms of effector secretion \& delivery, \& marker domains}

For T6SS-mediated deployment into recipient cells, effectors must be loaded onto the secreted tail that serves as their means of transportation across the cell envelope. The first delivery mechanism to be identified was the fusion of toxin domains to one of the T6SS tail components. Locating such effectors is relatively straightforward since it can be achieved by identifying tail components that possess varying C-terminal extensions. Another delivery mechanism is direct interaction of effectors with tail components (known as 'cargo effectors'). These interactions may be assisted by chaperone-like proteins called adapters. Identification of these effectors had so far been achieved by examining genes of unknown function within T6SS gene clusters, comparative proteomics and bioinformatics. Both delivery mechanisms often rely on the modular nature of effectors, as in many instances they are composed of a C-terminal toxin domain fused to an $\mathrm{N}$-terminal delivery or marker domain. These $\mathrm{N}$ - and $\mathrm{C}$-terminal domains are interchangeable, allowing for extraordinary diversification of effector repertoires in a 'lego-like' fashion (Figure 2).

\section{'Evolved' tail components}

The first T6SS-secreted protein that was demonstrated to function as an effector was the V. cholerae VgrG-1 containing a C-terminal actin cross-linking domain that targets eukaryotic cells [48]. In their work, Pukatzki et al. coined the term 'evolved VgrG' to describe VgrG proteins that contain varying C-terminal extensions [48]. Since this discovery, many 'evolved VgrGs' have been identified and shown to possess extended C-terminal domains with antibacterial activities, as in the case of $V$. cholerae VgrG-3 that harbors a C-terminal peptidoglycan-hydrolyzing toxin domain of the Tge superfamily [49]. $\mathrm{VgrG}$ is not the only tail component that can carry C-terminal extensions. The two other components, PAAR-containing protein and Hcp, were also shown to often be 'evolved' and fused to C-terminal antibacterial toxin domains with known or predicted activities, such as nuclease, phospholipase, NADase or pore forming [33,42,50,51]. Therefore, Hcp, VgrG and PAAR-containing proteins may also be regarded as T6SS-specific toxin delivery components. Notably, 'evolved Hcps' are mostly restricted to Enterobacteriaceae species [33].

'Evolved VgrG' and 'evolved PAAR' effectors can fit their C-terminal toxin domain extensions in the internal cavity that is formed between the T6SS spike and the baseplate complex [52]. However, it is less clear how 'evolved Hcp' effectors are loaded onto the tail. C-terminal extensions of 'evolved Hcp' effectors cannot protrude outside of the hexameric Hcp rings since this space is occupied by the sheath components Tss $\mathrm{B} / \mathrm{C}$ [53]. Therefore, such toxin domains likely reside in the inner cavity of the Hcp rings, where the $P$. aeruginosa effector Tse 2 was previously shown to reside [54]. However, since the inner diameter of Hcp rings is only approximately $40 \AA$ [54], it is probably too tight to fit six C-terminal toxin domains, suggesting that hexameric rings that include 'evolved Hcp' are heterogeneous and also include canonical, non 'evolved' Hcp. 


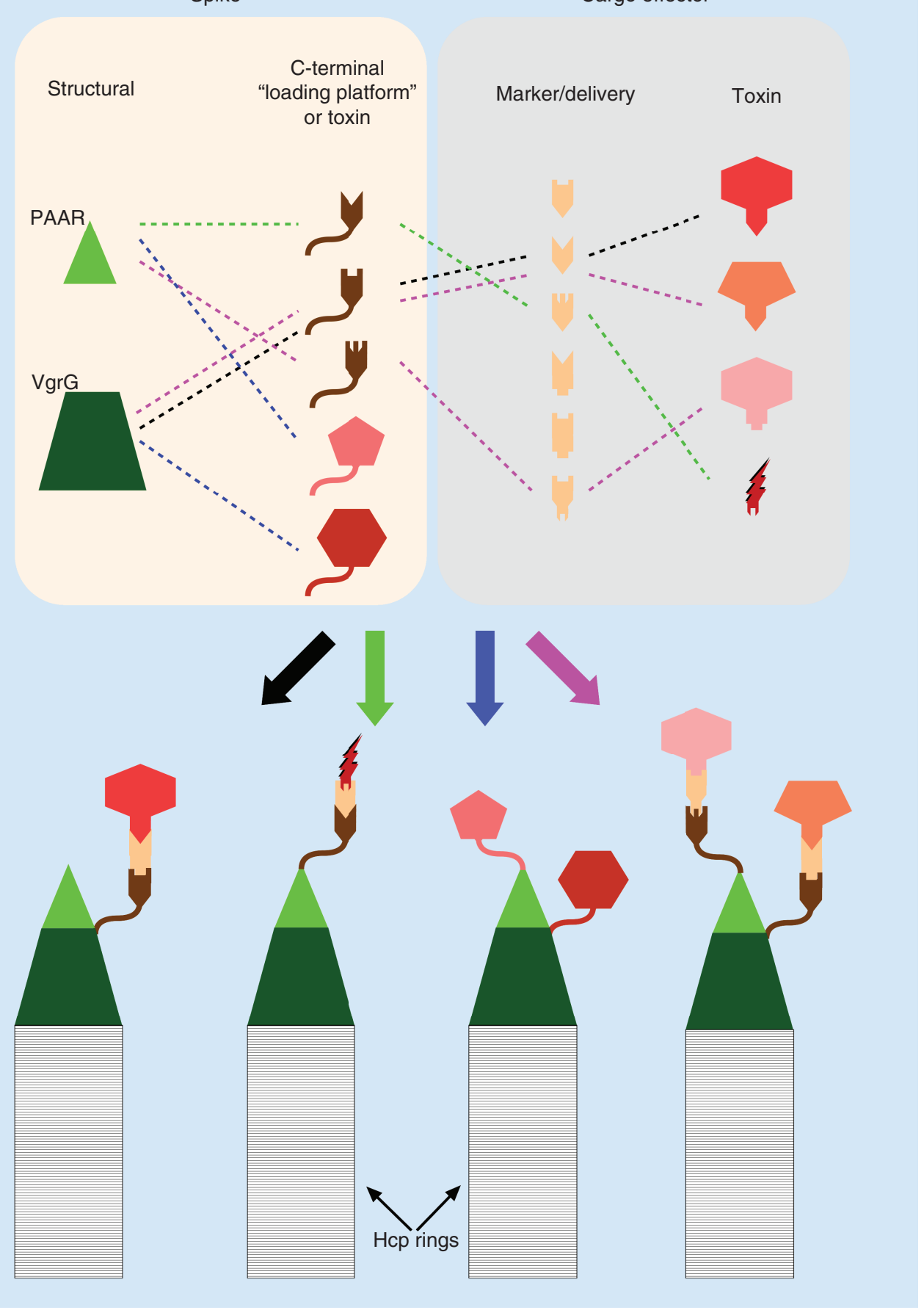

Figure 2. Type VI secretion system spikes are modular, toxic toolkits. Illustration of modular type VI secretion system tails made up of combinations of structural T6SS spike components (VgrG and PAAR-containing proteins) that can also carry various cargo effectors. Spike components may be fused to a C-terminal toxin domain or to domains that mediate effector binding ('loading platforms'). The latter may mediate delivery of a cargo effector, possibly via binding of a marker or delivery domain found in the cargo effector. The different combinations provide a dynamic arsenal that is loaded onto the Hcp tube to be fired at a recipient cell. 
'Evolved' tail components do not always contain a toxin domain in their C-terminal extensions. In fact, examination of common VgrG C-terminal extensions reveals that many contain domains of unknown function (DUFs) that are not predicted to exert toxic activity, such as DUF2345. Therefore, non-toxin C-terminal extensions of T6SS tail components may also serve as 'loading platforms' for effectors. This mechanism of effector loading onto the tube, which is based on specificity conferred by the C-terminus of an 'evolved VgrG', was demonstrated in several bacteria, such as E. coli [41], A. tumefaciens [55], P. aeruginosa [56] and Vibrio parahaemolyticus [28]. Indeed, VgrG proteins were suggested to serve as 'à la Carte' effector delivery platforms [57]. A similar observation was reported for 'evolved PAAR' proteins, many of which contain extensions with transthyretin (TTR) domains that are known to mediate protein-protein interactions, and can thus serve as 'loading platforms' for additional effectors [50,58]. Since many T6SSs are associated with multiple Hcp, VgrG or PAAR-containing protein variants, the different combination of these three core components may mediate the delivery of distinct effector arsenals by the same T6SS under different conditions (Figure 2) $[48,55,57,59,60]$.

\section{T6SS-associated marker domains}

The cocktail of toxin domains that decorate the secreted T6SS tail is further spiked by varying effectors that bind Hcp, VgrG or PAAR-containing proteins (i.e., 'cargo effectors'). While many of these effectors likely bind C-terminal extensions of 'evolved' tail components, as explained above, the mechanisms that enable them to locate and bind to the tail are largely unknown. Effectors that bind Hcp and reside inside the Hcp tube seem to be small proteins since they need to fit into a narrow space, and they do not appear to possess domains in addition to the toxin domain [54]. On the other hand, various effectors have been proposed to use VgrG or PAAR-containing proteins for their secretion $[39,41,55,57,59,61]$; these are usually larger proteins that often contain additional N-terminal domains that mark them as secreted effectors (hereafter, marker domains).

To date, two domains have been proposed as specific markers of T6SS effectors, MIX (Marker for type sIX effectors) [39] and FIX (Found in type sIX effector) [28]. Both MIX and FIX are found N-terminal to known or predicted toxin domains. Occasionally, they are fused to N-terminal VgrG or PAAR domain, further demonstrating their association with the T6SS. MIX and FIX are genetically linked to T6SSs, and are encoded almost exclusively in bacteria carrying T6SSs [28,39]. While many MIX-containing proteins have been demonstrated to be T6SS effectors [39,45,62,63], FIX had only been discovered recently, and few examples were experimentally reported [28]. Since many C-terminal regions fused to MIX and FIX are not similar to known toxins [27,28,39], it is plausible that they are novel toxin domains yet to be described. It remains unclear whether MIX and FIX serve as delivery domains per se, directing the toxins to the T6SS tail and mediating interaction with tail components, or whether they serve a structural or some other functional role in the effectors' mechanism of secretion, delivery into neighboring cell, or mechanism of action.

In addition to MIX and FIX, many T6SS effectors carry rearrangement hotspot (Rhs) repeats N-terminal to the toxin domain [30]. Therefore, Rhs can also be regarded as a marker for secreted effectors. However, since Rhs is not restricted to T6SS effectors [30,64], it is not strictly a T6SS marker. Structural analyses of Rhs-like toxins revealed that Rhs possibly encapsulates the toxin domain [65], suggesting that it plays a structural role in effector maturation rather than a functional role in T6SS-mediated secretion. Examination of sequences fused to T6SS effectors will surely reveal additional T6SS markers and delivery domains in the near future.

The described marker domains (MIX, FIX and Rhs), as well as the T6SS delivery domains and proteins (Hcp, VgrG and PAAR-containing protein) can be used to identify new T6SS effectors and thus new toxin domains located at their C-termini. Indeed, many $\mathrm{C}$-terminal domains fused to these $\mathrm{N}$-terminal marker and delivery domains have been proposed as toxins. Their predicted or hypothetical activities range from pore-forming toxins to nucleases, proteases or other types of hydrolases $[27,28,33,39,48]$. Noteworthy, similar toxin domains to those identified in many T6SS effectors are also found in effectors of other systems implicated in interbacterial antagonism $[4,17,28,30,66,67]$. Therefore, deciphering the activity of a new toxin family could have implications relevant to more than one interbacterial competition mechanism, or even to nonsecreted toxin-antitoxin modules, which are widespread in bacteria and may use similar antibacterial mechanisms [68].

\section{Adapters \& chaperones}

Some T6SS 'cargo effectors' seem to require additional assistance when they are being loaded onto the T6SS tail. The aid is provided by proteins referred to as adapters or chaperones. The first protein that was shown to function as a chaperone for T6SS effectors was the tube component Hcp. In P. aeruginosa, the presence of Hcp was shown 
to be required for the stability of effectors that bind to it and use it as their 'loading platform', since deletion or depletion of Hcp resulted in reduced levels of intracellular effector accumulation [54].

In addition to Hcp, which is a central structural component that is required for T6SS activity, three other protein families were described as T6SS adapters: DUF4123 [69,70], DUF1795 (also referred to as Eag) [31,59,71] and DUF2169 [55]. These adapters are usually encoded upstream of their cognate effectors, they are not required for T6SS activity, and they are not secreted by T6SS. Rather, they probably serve to stabilize effectors and aid in their loading onto the tail, and thus they are required for the T6SS-mediated delivery of their cognate effector. Adapters were shown to physically interact with their cognate effectors, yet it remains largely unknown whether the three known adapter families function via similar mechanisms, whether they bind similar structural elements within T6SS effectors (e.g., Eags were shown to bind and shield transmembrane helices found in T6SS effectors [71]), or whether they functionally contribute to the loading of effectors onto the T6SS tail. An additional mechanism of effector delivery was recently reported, in which the DUF 4123 adapter was aided by a co-adapter that was encoded between the adapter and the effector gene [58]. Importantly, these T6SS-specific adapters can be used to indirectly identify adjacently encoded genes as T6SS effectors [70].

\section{Dynamic T6SS effector repertoires \& mobility of effectors}

Comparisons of bacterial genomes harboring antibacterial T6SSs revealed high level of divergence in effector repertoires. Even isolates of the same bacterial species were found to harbor diverse effector repertoires, sometimes encoding different effectors in the same genomic synteny $[39,69,72,73]$. These observations raised the possibility that the T6SS effector repertoire in a given bacterial strain is dynamic and subject to change, possibly via horizontal gene transfer. Indeed, correlative evidence of horizontal mobility of T6SS effectors was reported in various vibrios. The presence of MIX effectors adjacent to, or in mobile genetic elements such as transposons or prophages [62,74], together with evidence of recombination sites within DUF4123 adapters [69] and of sequential acquisition of effector and immunity pairs [75], supported the concept of dynamic horizontal exchange of T6SS effectors between bacteria. In 2015, Borgeaud et al. [76] demonstrated that antibacterial T6SS can foster horizontal gene transfer in $V$. cholerae, thus raising the possibility that T6SS effectors can be horizontally shared between competing bacteria in a T6SS-mediated manner. This effector arsenal diversification mechanism was later confirmed by Thomas et al. [77], who demonstrated horizontal acquisition of T6SS effectors by $V$. cholerae via natural transformation. Since bacteria that acquire such new effectors gain competitive advantage over their parental kin, it is likely that the dynamics of effector acquisition play a role in shaping the composition of bacterial communities $[62,77,78]$.

\section{Future perspective}

T6SS is a widespread machine used by Gram-negative bacteria to outcompete rivals. While we have witnessed considerable progress in recent years toward understanding the roles of T6SSs, their structural and dynamic aspects, and even discovered many of their delivered effectors, much remains unknown. Most of our current knowledge comes from studying a handful of culturable and genetically tractable bacterial species, whereas the functions and effectors of T6SSs in other bacteria remain speculative. In many cases, we still do not understand under what conditions are T6SSs activated, what is their effector repertoire, or how to determine whether a certain T6SS mediates antibacterial activities, virulence activities, or both.

The ability to activate T6SSs in bacteria is required to study their functions and to determine their effector repertoires. Even though we can identify T6SS effectors computationally using delivery or marker domains, or by identifying potential neighboring adapters, many bacteria that harbor T6SSs do not have identifiable effectors. Identifying potential effectors in these bacteria will thus either require the employment of unbiased strategies such as comparative proteomics, which will also necessitate an active T6SS, or the discovery of new marker domains that can reveal additional novel T6SS effectors.

As discussed above, the study of individual effectors is useful to decipher antibacterial mechanisms of action. Yet, considering the function or activity of individual effectors may represent a very simplistic and reductionist point of view. As work by LaCourse et al. recently underlined [38], we also need to take into account the role and function of T6SS effectors in the context of diverse effector repertoires, and we should consider that such repertoires are employed under constantly changing environmental conditions. Therefore, it is expected that additional work considering the potential synergism between effectors will soon emerge. Of note, the possibility of potential synergism between T6SS effectors is not limited to effectors delivered by a single system. Since many bacteria harbor multiple T6SSs [79], each with its own effector arsenal, it is also plausible that synergism between 
T6SSs with different effector repertoires will be revealed. Therefore, one must consider the effector repertoires of individual T6SSs as well as the whole T6SS effector repertoire of a bacterial isolate. A comprehensive database of T6SS effectors that makes use of our knowledge on delivery, marker, toxin and adapter domains will surely be instrumental in such future endeavors.

\section{Conclusion}

Last, one must consider the enormous potential found in T6SSs as natural antibacterial mechanisms. These systems, either in their entirety or just as single effectors with antibacterial activities, provide a lucrative pool that can be exploited to develop novel antibacterial treatment strategies. This potential underscores the importance of continued research into T6SSs and their effectors.

\section{Executive summary}

Antibacterial type VI secretion system effectors: activities \& targets

- Type VI secretion systems (T6SSs) can deliver multiple effectors with diverse targets into competing cells. Antibacterial effectors are known to target nucleic acids, membranes, cell wall, energy balance and specific essential proteins.

- Several T6SS effectors are trans-kingdom toxins that target not only competing bacteria, but also eukaryotic cells.

- Effectors can act synergistically inside target cells, and their activities may depend on external conditions.

Mechanisms of effector secretion \& delivery, \& marker domains

- The tube and spike components Hcp, VgrG and PAAR-containing protein may be regarded as T6SS-specific toxin delivery components that can carry C-terminal toxin domain extensions.

- Non-toxin C-terminal extensions of T6SS tail components may also serve as 'loading platforms' for 'cargo effectors'.

- Large modular effectors use VgrG or PAAR-containing proteins for their secretion, and often contain N-terminal domains such as MIX, FIX or Rhs.

- Some T6SS 'cargo effectors' require adapters for proper T6SS loading and delivery.

Dynamic T6SS effector repertoires \& mobility of effectors

- Horizontal acquisition of T6SS effectors diversifies T6SS effector repertoires, even between isolates of the same bacterial species. Such dynamic effector acquisition may play a role in shaping the composition of bacterial communities.

Future perspective

- T6SSs and their effectors carry enormous potential as natural antibacterial mechanisms. They provide a lucrative pool that can be exploited to develop novel antibacterial treatment strategies.

\section{Author contributions}

B Jana and D Salomon wrote the manuscript.

\section{Acknowledgments}

The authors thank members of the Salomon Lab for helpful discussions. The authors apologize to the colleagues who have made important contributions to the field but whose work was not discussed or cited here due to topic and length constraints.

Financial \& competing interests disclosure

D Salomon is funded by the European Research Council under the EU's Horizon 2020 research and innovation program (grant agreement No. 714224), and by Israel Science Foundation (grant no. 920/17). D Salomon is an Alon Fellow. The funders played no role in the study design, data collection, and analysis, as well as the decision to publish or in preparing the manuscript. The authors have no other relevant affiliations or financial involvement with any organization or entity with a financial interest in or financial conflict with the subject matter or materials discussed in the manuscript apart from those disclosed.

No writing assistance was utilized in the production of this manuscript.

\section{Open access}

This work is licensed under the Attribution-NonCommercial-NoDerivatives 4.0 Unported License. To view a copy of this license, visit http://creativecommons.org/licenses/by-nc-nd/4.0/ 


\section{References}

Papers of special note have been highlighted as: $\bullet$ of interest; $\bullet \bullet$ of considerable interest

1. Nadell CD, Drescher K, Foster KR. Spatial structure, cooperation and competition in biofilms. Nat. Rev. Microbiol. 14(9), 589-600 (2016).

2. Cascales E, Buchanan SK, Duché D et al. Colicin biology. Microbiol. Mol. Biol. Rev. 71(1), 158-229 (2007).

3. García-Bayona L, Guo MS, Laub MT. Contact-dependent killing by Caulobacter crescentus via cell surface-associated, glycine zipper proteins. Elife 6, e24869 (2017).

4. Aoki SK, Diner EJ, de Roodenbeke C t'Kint et al. A widespread family of polymorphic contact-dependent toxin delivery systems in bacteria. Nature 468(7322), 439-442 (2010).

5. Pukatzki S, Ma AT, Sturtevant D et al. Identification of a conserved bacterial protein secretion system in Vibrio cholerae using the Dictyostelium host model system. Proc. Natl Acad. Sci. 103(5), 1528-1533 (2006).

-. The first report coining the name type VI secretion system as a protein delivery machine in Vibrio cholerae.

6. Mougous JD, Cuff ME, Raunser S et al. A virulence locus of Psendomonas aeruginosa encodes a protein secretion apparatus. Science (80) 312(5779), 1526-1530 (2006).

7. Ghequire MGK, De Mot R. The tailocin tale: peeling off phage tails. Trends Microbiol. 23(10), 587-590 (2015).

8. Cao Z, Casabona MG, Kneuper H, Chalmers JD, Palmer T. The type VII secretion system of Staphylococcus aureus secretes a nuclease toxin that targets competitor bacteria. Nat. Microbiol. 2(1), 16183 (2016).

9. Souza DP, Oka GU, Alvarez-Martinez CE et al. Bacterial killing via a type IV secretion system. Nat. Commun. 6(1), 6453 (2015).

10. Bingle LE, Bailey CM, Pallen MJ. Type VI secretion: a beginner's guide. Curr. Opin. Microbiol. 11(1), 3-8 (2008).

11. Nguyen VS, Douzi B, Durand E, Roussel A, Cascales E, Cambillau C. Towards a complete structural deciphering of type VI secretion system. Curr. Opin. Struct. Biol. 49, 77-84 (2018).

12. Bladergroen MR, Badelt K, Spaink HP. Infection-blocking genes of a symbiotic Rhizobium leguminosarum strain that are involved in temperature-dependent protein secretion. Mol. Plant-Microbe Interact. 16(1), 53-64 (2003).

13. Srinivasa Rao PS, Yamada Y, Tan YP, Leung KY. Use of proteomics to identify novel virulence determinants that are required for Edwardsiella tarda pathogenesis. Mol. Microbiol. 53(2), 573-586 (2004).

14. Hood RD, Singh P, Hsu FS et al. A type VI secretion system of Pseudomonas aeruginosa targets a toxin to bacteria. Cell Host Microbe 7(1), 25-37 (2010).

-. The first report of type VI secretion system mediating interbacterial competition and the discovery of the first antibacterial effectors.

15. Russell AB, Singh P, Brittnacher M et al. A widespread bacterial type VI secretion effector superfamily identified using a heuristic approach. Cell Host Microbe 11(5), 538-549 (2012).

- Describes the identification of a widespread superfamily of type VI secretion system effectors that target the peptidoglycan.

16. Ma J, Sun M, Dong W, Pan Z, Lu C, Yao H. PAAR-Rhs proteins harbor various C-terminal toxins to diversify the antibacterial pathways of type VI secretion systems. Environ. Microbiol. 19(1), 345-360 (2017).

17. Zhang D, de Souza RF, Anantharaman V, Iyer LM, Aravind L. Polymorphic toxin systems: comprehensive characterization of trafficking modes, processing, mechanisms of action, immunity and ecology using comparative genomics. Biol. Direct. 7, 18 (2012).

18. Callewaert L, Michiels CW. Lysozymes in the animal kingdom. J. Biosci. 35(1), 127-160 (2010).

19. Vollmer W, Blanot D, De Pedro MA. Peptidoglycan structure and architecture. FEMS Microbiol. Rev. 32(2), 149-167 (2008).

20. Russell AB, Hood RD, Bui NK, Leroux M, Vollmer W, Mougous JD. Type VI secretion delivers bacteriolytic effectors to target cells. Nature 475(7356), 343-349 (2011).

21. Rigden DJ, Jedrzejas MJ, Galperin MY. Amidase domains from bacterial and phage autolysins define a family of $\gamma$-d,l-glutamate-specific amidohydrolases. Trends Biochem. Sci. 28(5), 230-234 (2003).

22. Ma J, Sun M, Pan Z, Lu C, Yao H. Diverse toxic effectors are harbored by VgrG islands for interbacterial antagonism in type VI secretion system. Biochim. Biophys. Acta Gen. Subj. 1862(7), 1635-1643 (2018).

23. English G, Trunk K, Rao VA, Srikannathasan V, Hunter WN, Coulthurst SJ. New secreted toxins and immunity proteins encoded within the type VI secretion system gene cluster of Serratia marcescens. Mol. Microbiol. 86(4), 921-936 (2012).

24. Srikannathasan V, English G, Bui NK et al. Structural basis for type VI secreted peptidoglycan DL-endopeptidase function, specificity and neutralization in Serratia marcescens. Acta Crystallogr. Sect. D Biol. Crystallogr. 69(12), 2468-2482 (2013).

25. Whitney JC, Chou S, Russell AB et al. Identification, structure, and function of a novel type VI secretion peptidoglycan glycoside hydrolase effector-immunity pair. J. Biol. Chem. 288(37), 26616-26624 (2013).

26. Zhang D, Iyer LM, Aravind L. A novel immunity system for bacterial nucleic acid degrading toxins and its recruitment in various eukaryotic and DNA viral systems. Nucleic Acids Res. 39(11), 4532-4552 (2011). 
27. Dar Y, Salomon D, Bosis E. The antibacterial and anti-eukaryotic type VI secretion system MIX-effector repertoire in vibrionaceae. Mar. Drugs 16(11), 433 (2018).

28. Jana B, Fridman CM, Bosis E, Salomon D. A modular effector with a DNase domain and a marker for T6SS substrates. Nat. Commun. 10(1), 3595 (2019).

- Describes FIX (found in type six effector) as a type VI secretion system marker domain, and the PoNe DNase toxin family.

29. Ma LS, Hachani A, Lin JS, Filloux A, Lai EM. Agrobacterium tumefaciens deploys a superfamily of type VI secretion DNase effectors as weapons for interbacterial competition in planta. Cell Host Microbe 16(1), 94-104 (2014).

30. Koskiniemi S, Lamoureux JG, Nikolakakis KC et al. Rhs proteins from diverse bacteria mediate intercellular competition. Proc. Natl Acad. Sci. USA 110(17), 7032-7037 (2013).

- Discovery of Rhs as a marker for secreted toxins.

31. Alcoforado Diniz J, Coulthurst SJ. Intraspecies competition in Serratia marcescens is mediated by type VI-secreted Rhs effectors and a conserved effector-associated accessory protein. J. Bacteriol. 197(14), 2350-2360 (2015).

32. Fitzsimons TC, Lewis JM, Wright A et al. Identification of novel Acinetobacter baumannii type VI secretion system antibacterial effector and immunity pairs. Infect. Immun. 86(8), e00297-e00318 (2018).

33. Ma J, Pan Z, Huang J, Sun M, Lu C, Yao H. The Hcp proteins fused with diverse extended-toxin domains represent a novel pattern of antibacterial effectors in type VI secretion systems. Virulence 8(7), 1189-1202 (2017).

34. Pissaridou P, Allsopp LP, Wettstadt S, Howard SA, Mavridou DAI, Filloux A. The Pseudomonas aeruginosa T6 SS-VgrG1b spike is topped by a PAAR protein eliciting DNA damage to bacterial competitors. Proc. Natl Acad. Sci. USA 115(49), 12519-12524 (2018).

35. Steczkiewicz K, Muszewska A, Knizewski L, Rychlewski L, Ginalski K. Sequence, structure and functional diversity of PD-(D/E)XK phosphodiesterase superfamily. Nucleic Acids Res. 40(15), 7016-7045 (2012).

36. Miyata ST, Unterweger D, Rudko SP, Pukatzki S. Dual expression profile of type VI secretion system immunity genes protects pandemic Vibrio cholerae. PLoS Pathog. 9(12), 1-18 (2013).

37. Iacovache I, van der Goot FG, Pernot L. Pore formation: an ancient yet complex form of attack. Biochim. Biophys. Acta Biomembr. 1778(7-8), 1611-1623 (2008).

38. LaCourse KD, Peterson SB, Kulasekara HD, Radey MC, Kim J, Mougous JD. Conditional toxicity and synergy drive diversity among antibacterial effectors. Nat. Microbiol. 3(4), 440-446 (2018).

- Introduces the concepts of effector efficacy and synergism in type VI secretion systems.

39. Salomon D, Kinch LN, Trudgian DC et al. Marker for type VI secretion system effectors. Proc. Natl Acad. Sci. USA 111(25), 9271-9276 (2014).

-• Describes MIX (marker for type six effectors) as the first nonstructural domain that marks proteins as type VI secretion system effectors.

40. Russell AB, Leroux M, Hathazi K et al. Diverse type VI secretion phospholipases are functionally plastic antibacterial effectors. Nature 496(7446), 508-512 (2013).

41. Flaugnatti N, Le TTH, Canaan $\mathrm{S}$ et al. A phospholipase $\mathrm{A}_{1}$ antibacterial type VI secretion effector interacts directly with the C-terminal domain of the VgrG spike protein for delivery. Mol. Microbiol. 99(6), 1099-1118 (2016).

42. Whitney JC, Quentin D, Sawai $\mathrm{S}$ et al. An interbacterial $\mathrm{NAD}(\mathrm{P})^{+}$glycohydrolase toxin requires elongation factor Tu for delivery to target cells. Cell 163(3), 607-619 (2015).

43. Tang JY, Bullen NP, Ahmad S, Whitney JC. Diverse NADase effector families mediate interbacterial antagonism via the type VI secretion system. J. Biol. Chem. 293(5), 1504-1514 (2018).

44. Ting S-Y, Bosch DE, Mangiameli SM et al. Bifunctional immunity proteins protect bacteria against FtsZ-targeting ADP-ribosylating toxins. Cell 175(5), 1380-1392 (2018).

45. Miyata ST, Kitaoka M, Brooks TM, McAuley SB, Pukatzki S. Vibrio cholerae requires the type VI secretion system virulence factor VasXx to kill Dictyostelium discoideum. Infect. Immun. 79(7), 2941-2949 (2011).

46. Jiang F, Waterfield NR, Yang J, Yang G, Jin Q. A Pseudomonas aeruginosa type VI secretion phospholipase D effector targets both prokaryotic and eukaryotic cells. Cell Host Microbe 15(5), 600-610 (2014).

47. Jiang F, Wang X, Wang B et al. The Pseudomonas aeruginosa type VI secretion PGAP1-like effector induces host autophagy by activating endoplasmic reticulum stress. Cell Rep. 16(6), 1502-1509 (2016).

48. Pukatzki S, Ma AT, Revel AT, Sturtevant D, Mekalanos JJ. Type VI secretion system translocates a phage tail spike-like protein into target cells where it cross-links actin. Proc. Natl Acad. Sci. USA 104(39), 15508-15513 (2007).

-• Introduces the concept of 'evolved VgrG' as type VI secretion system effectors.

49. Brooks TM, Unterweger D, Bachmann V, Kostiuk B, Pukatzki S. Lytic activity of the Vibrio cholerae type VI secretion toxin VgrG-3 is inhibited by the antitoxin TsaB. J. Biol. Chem. 288(11), 7618-7625 (2013).

50. Shneider MM, Buth SA, Ho BT, Basler M, Mekalanos JJ, Leiman PG. PAAR-repeat proteins sharpen and diversify the type VI secretion system spike. Nature 500(7462), 350-353 (2013). 
51. Blondel CJ, Jiménez JC, Contreras I, Santiviago CA. Comparative genomic analysis uncovers 3 novel loci encoding type six secretion systems differentially distributed in Salmonella serotypes. BMC Genomics 10(1), 354 (2009).

52. Nazarov S, Schneider JP, Brackmann M, Goldie KN, Stahlberg H, Basler M. Cryo-EM reconstruction of type VI secretion system baseplate and sheath distal end. EMBO J. 37(4), e201797103 (2017).

53. Wang J, Brackmann M, Castaño-Díez D et al. Cryo-EM structure of the extended type VI secretion system sheath-tube complex. Nat. Microbiol. 2(11), 1507-1512 (2017).

54. Silverman JM, Agnello DM, Zheng $\mathrm{H}$ et al. Haemolysin coregulated protein is an exported receptor and chaperone of type VI secretion substrates. Mol. Cell. 51(5), 584-593 (2013).

55. Bondage DD, Lin J-S, Ma L-S, Kuo C-H, Lai E-M. VgrG C terminus confers the type VI effector transport specificity and is required for binding with PAAR and adaptor-effector complex. Proc. Natl Acad. Sci. USA 113(27), E3931-E3940 (2016).

56. Wettstadt S, Wood TE, Fecht S, Filloux A. Delivery of the Pseudomonas aeruginosa phospholipase effectors PldA and PldB in a VgrGand H2-T6SS-dependent manner. Front. Microbiol. 10, 1718 (2019).

57. Hachani A, Allsopp LP, Oduko Y, Filloux A. The VgrG proteins are 'à la carte’ delivery systems for bacterial type VI effectors. J. Biol. Chem. 289(25), 17872-17884 (2014).

58. Burkinshaw BJ, Liang X, Wong M, Le ANH, Lam L, Dong TG. A type VI secretion system effector delivery mechanism dependent on PAAR and a chaperone-co-chaperone complex. Nat. Microbiol. 3(5), 632-640 (2018).

59. Cianfanelli FR, Alcoforado Diniz J, Guo M, De Cesare V, Trost M, Coulthurst SJ. VgrG and PAAR proteins define distinct versions of a functional type VI secretion system. PLoS Pathog. 12(6), 1-27 (2016).

60. Wood TE, Howard SA, Wettstadt S, Filloux A. PAAR proteins act as the 'sorting hat' of the type VI secretion system. Microbiology 165(11) (2019).

61. Bernal P, Allsopp LP, Filloux A, Llamas MA. The Pseudomonas putida T6SS is a plant warden against phytopathogens. ISME J. 11(4), 972-987 (2017).

62. Salomon D, Klimko JA, Trudgian DC et al. Type VI secretion system toxins horizontally shared between marine bacteria. PLoS Pathog. 11(8), 1-20 (2015).

63. Ray A, Schwartz N, de Souza Santos M, Zhang J, Orth K, Salomon D. Type VI secretion system MIX-effectors carry both antibacterial and anti-eukaryotic activities. EMBO Rep. 18(11), e201744226 (2017).

64. Poole SJ, Diner EJ, Aoki SK et al. Identification of functional toxin/immunity genes linked to contact-dependent growth inhibition (CDI) and rearrangement hotspot (Rhs) systems. PLoS Genet. 7(8), e1002217 (2011).

65. Busby JN, Panjikar S, Landsberg MJ, Hurst MRH, Lott JS. The BC component of ABC toxins is an RHS-repeat-containing protein encapsulation device. Nature 501(7468), 547-550 (2013).

66. Whitney JC, Peterson SB, Kim J et al. A broadly distributed toxin family mediates contact-dependent antagonism between Gram-positive bacteria. Elife 6, e26938 (2017).

67. Jamet A, Nassif X. New players in the toxin field: polymorphic toxin systems in bacteria. MBio 6(3), e00285-e00315 (2015).

68. Yamaguchi Y, Park J-H, Inouye M. Toxin-antitoxin systems in bacteria and archaea. Annu. Rev. Genet. 45(1), 61-79 (2011).

69. Unterweger D, Kostiuk B, Ötjengerdes R, Wilton A, Diaz-Satizabal L, Pukatzki S. Chimeric adaptor proteins translocate diverse type VI secretion system effectors in Vibrio cholerae. EMBO J. 34(16), 2198-2210 (2015).

70. Liang X, Moore R, Wilton M, Wong MJQ, Lam L, Dong TG. Identification of divergent type VI secretion effectors using a conserved chaperone domain. Proc. Natl Acad. Sci. USA 112(29), 9106-9111 (2015).

71. Quentin D, Ahmad S, Shanthamoorthy P, Mougous JD, Whitney JC, Raunser S. Mechanism of loading and translocation of type VI secretion system effector Tse6. Nat. Microbiol. 3(10), 1142-1152 (2018).

72. Unterweger D, Miyata ST, Bachmann V et al. The Vibrio cholerae type VI secretion system employs diverse effector modules for intraspecific competition. Nat. Commun. 5(1), 3549 (2014).

73. Alteri CJ, Himpsl SD, Zhu K et al. Subtle variation within conserved effector operon gene products contributes to T6SS-mediated killing and immunity. PLoS Pathog. 13(11), 1-22 (2017).

74. Li P, Kinch LN, Ray A et al. Acute hepatopancreatic necrosis disease-causing Vibrio parahaemolyticus strains maintain an antibacterial type VI secretion system with versatile effector repertoires. Appl. Environ. Microbiol. 83(13), e00737-e00817 (2017).

75. Kirchberger PC, Unterweger D, Provenzano D, Pukatzki S, Boucher Y. Sequential displacement of type VI secretion system effector genes leads to evolution of diverse immunity gene arrays in Vibrio cholerae. Sci. Rep. 7(February), 1-12 (2017).

76. Borgeaud S, Metzger LC, Scrignari T, Blokesch M. The type VI secretion system of Vibrio cholerae fosters horizontal gene transfer. Science 347(6217), 63-67 (2015).

-. Shows that the antibacterial type VI secretion system and the DNA uptake machinery are co-regulated in Vibrio cholerae.

77. Thomas J, Watve SS, Ratcliff WC, Hammer BK. Horizontal gene transfer of functional type VI killing genes by natural transformation. MBio 8(4), 1-11 (2017). 
78. Salomon D. MIX and match: mobile T6SS MIX-effectors enhance bacterial fitness. Mob. Genet. Elements 6(1), e1123796 (2016).

79. Boyer F, Fichant G, Berthod J, Vandenbrouck Y, Attree I. Dissecting the bacterial type VI secretion system by a genome wide in silico analysis: what can be learned from available microbial genomic resources? BMC Genomics 10 (2009). 
